\title{
Phenylamine-Functionalized Graphene-Copper Composites with High Thermal Conductivity: Implications for Thermal Dissipation
}

\author{
Xiaofang $\mathrm{Li}^{\dagger,}$, , Meng $\mathrm{An}^{\dagger}$, Peide $\mathrm{Li}^{\dagger}$, Jianwen Miu ${ }^{\dagger}$, Qiang $\mathrm{Wu}^{\dagger}$, Fenghua Zheng ${ }^{\dagger}, *$, \\ Juantao Jiang ${ }^{\dagger}{ }^{*}$, Hongqiang Wang ${ }^{\dagger}$, Youguo Huang ${ }^{\dagger}$, and Qingyu $\mathrm{Li}^{\dagger}$ \\ School of Chemistry and Pharmaceutical Sciences, Guangxi Key Laboratory of Low Carbon \\ Energy Materials, Guangxi Normal University, Guilin, 541004, China \\ \$ School of Food and Chemical Engineering, Shaoyang University, Shaoyang, 422000, China \\ *E-mail: zhengfh870627@163.com (F. Zheng); jtjiang@gxnu.edu.cn (J. Jiang)
}

\section{Detailed Preparation of Phenylamine-Functionalized Graphene}

$100 \mathrm{mg}$ graphene was dispersed into $150 \mathrm{~mL}$ ethanol and then stirred for $10 \mathrm{~min}$ with magneton followed by ultrasonic dispersion for $40 \mathrm{~min}$. The resulting suspension was added into $100 \mathrm{~mL}$ ethanol dissolved with $1.2872 \mathrm{~g}$ of PPD and then bubbled with $\mathrm{N}_{2}$ for $20 \mathrm{~min}$. Then, $4 \mathrm{~mL}$ isoamyl nitrite was added dropwise into the above suspension followed by vigorously magnetic stir at $80{ }^{\circ} \mathrm{C}$ for $16 \mathrm{~h}$ under reflux. After being cooled to room temperature, the suspension was then purified through a PTFE membrane with a pore diameter of $0.45 \mu \mathrm{m}$. Finally, the product was washed with ethanol until the filtrate becomes colorless to remove the excess unreacted PPD and isoamyl nitrite and then dried under vacuum at $80{ }^{\circ} \mathrm{C}$ for $4 \mathrm{~h}$. 


\section{Detailed Preparation of PA-Gr-Cu Composite}

PA-Gr-Cu composite was prepared by pulsed-current electrodeposition (PCE) process in an electrolytic bath consisting of $0.3 \mathrm{~mol} \mathrm{~L}^{-1} \mathrm{CuSO}_{4}$ and $0.7 \mathrm{~mol} \mathrm{~L}^{-1} \mathrm{H}_{2} \mathrm{SO}_{4}$ and desired concentration of PA-Gr. Copper with a size of $\Phi 10 \mathrm{~mm} \times 2 \mathrm{~mm}$ was used as the substrate. The copper was treated in acetone and ethanol for $20 \mathrm{~min}$ to remove the oil contaminations, and then immersed into a homemade polishing solution for 30 $\mathrm{s}$ and sequentially cleaned with pure water. Pure copper was adopted as a counter electrode to maintain the copper ion concentration in the electrolyte solution. The electrolyte solution was sonicated for $20 \mathrm{~min}$ before PCE and stirred with a magnetic stirrer during PCE process. The resulting composite was rinsed with pure water and absolute ethanol successively and then dried by an infrared lamp. The final composite was denoted as PA-Gr-Cu composite. For comparison, pristine graphene was also employed to prepare $\mathrm{Gr}-\mathrm{Cu}$ with the same PCE parameters.
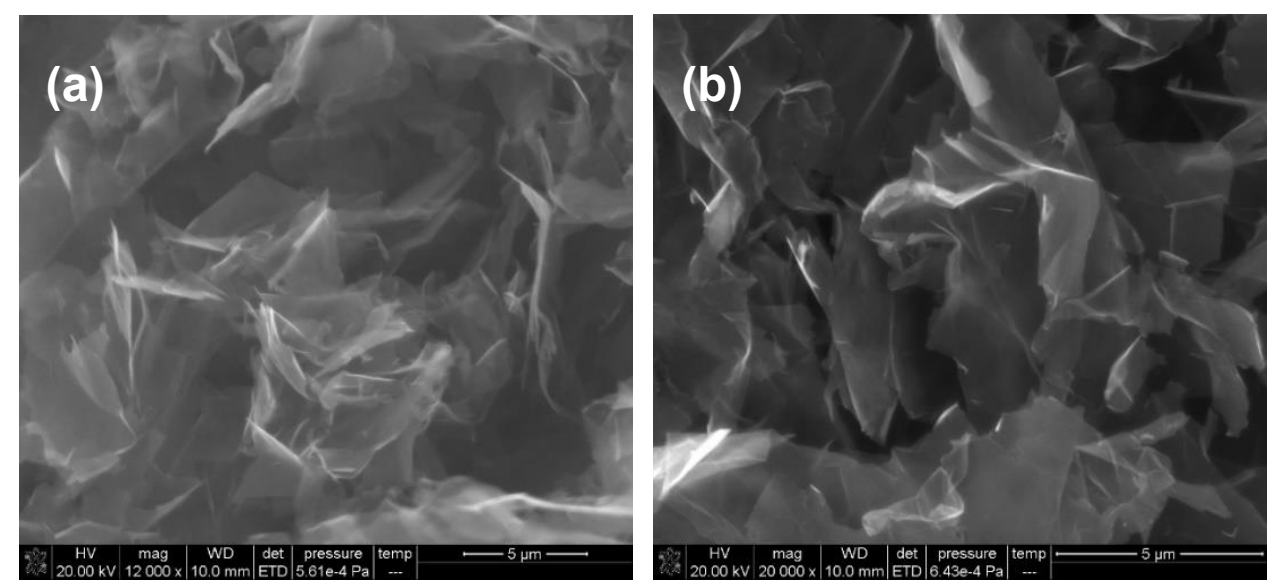

Figure S1. SEM of (a) Gr and (b) PA-Gr. 


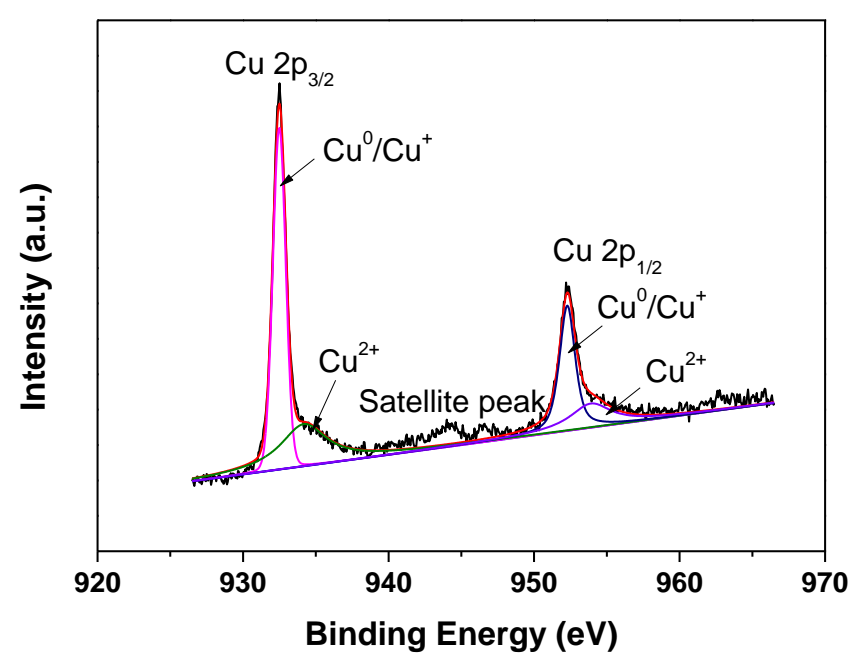

Figure S2. (a) XPS spectrum of $\mathrm{Cu} 2 \mathrm{p}$ of PA-Gr-Cu composite film. 

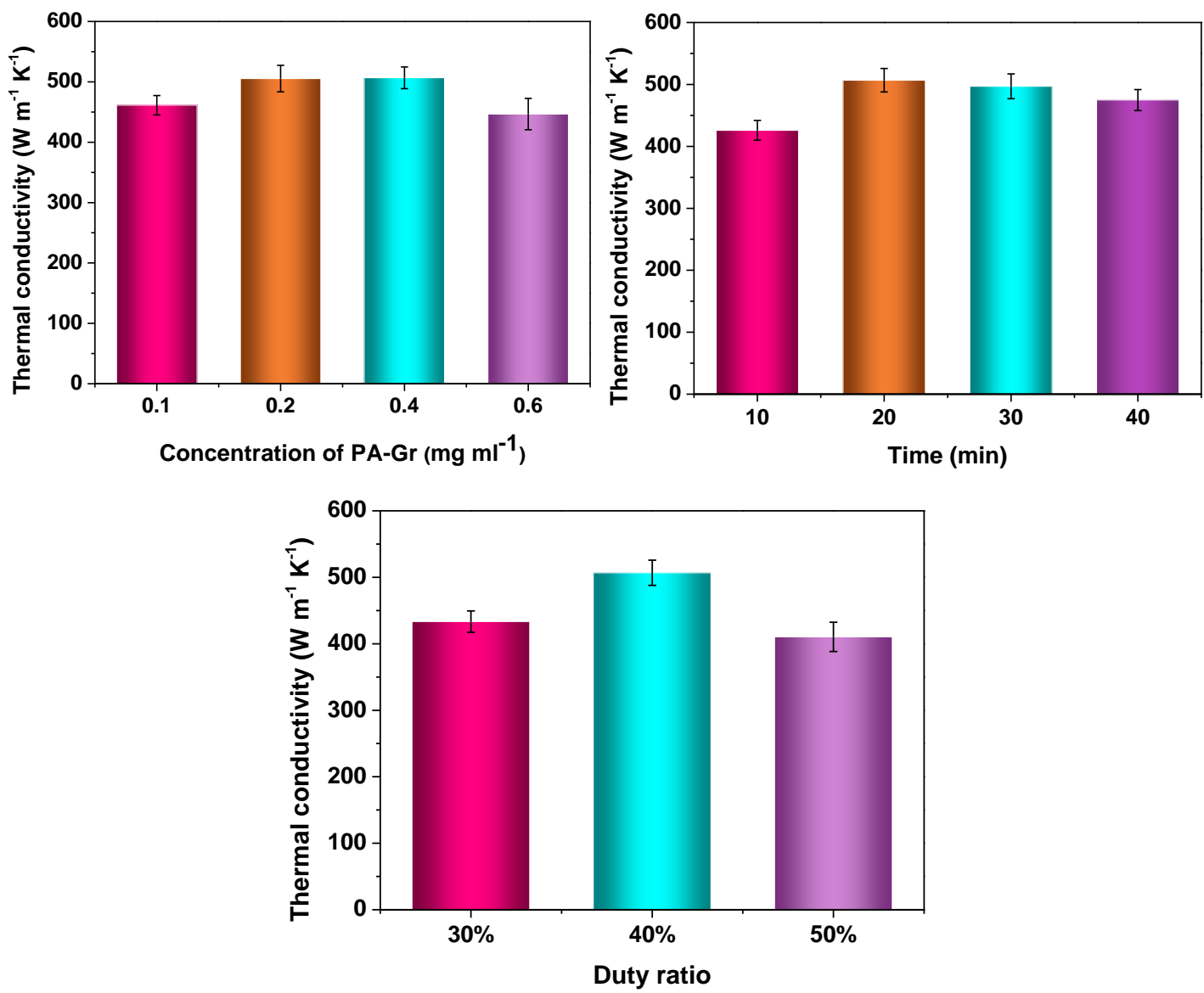

Figure S3. Effects of (a) PA-Gr concentration, (b) PCE time, and (c) duty ratio on TC. 